\title{
Constructions of quantum MDS codes
}

\author{
Hualu Liu* \\ School of Science, \\ Hubei University of Technology \\ Wuhan, Hubei 430068, China, \\ Email: hwlulu@aliyun.com \\ Xiusheng Liu \\ School of Mathematics and Physics, \\ Hubei Polytechnic University \\ Huangshi, Hubei 435003, China, \\ Email: Ixs6682@163.com
}

February 17, 2020

\begin{abstract}
Let $\mathbb{F}_{q}$ be a finite field with $q=p^{e}$ elements, where $p$ is a prime number and $e \geq 1$ is an integer. In this paper, by means of generalized Reed-Solomon (GRS) codes, we construct two new classes of quantum maximum-distance-separable ( quantum MDS) codes with parameters

$$
[[q+1,2 k-q-1, q-k+2]]_{q}
$$

for $\left\lceil\frac{q+2}{2}\right\rceil \leq k \leq q+1$, and

$$
[[n, 2 k-n, n-k+1]]_{q}
$$

for $n \leq q$ and $\left\lceil\frac{n}{2}\right\rceil \leq k \leq n$. Our constructions improve and generalize some results of available in the literature. Moreover, we give an affirmative answer to the open problem proposed by Fang et al. in [13].
\end{abstract}

Key Words: $s$-Galois dual codes; quantum MDS codes; GRS codes

${ }^{*}$ Corresponding author. 


\section{Introduction}

Quantum error correction codes play an important role in the fields of fighting against decoherence and quantum noise. It is well-known that a class of quantum error correction codes is obtained from the Calderbank-Shor-Steane (CSS) construction [6]. According to [7], a CSS quantum code is succinctly represented as a pair of linear codes $\left(C_{1}, C_{2}\right)$ over finite fields $\mathbb{F}_{p^{e}}$ with $C_{2} \subset C_{1}$, where $p$ is a prime and $e$ is a positive integer. Note that a CSS quantum code is a Hilbert space associated with a code pair $\left(C_{1}, C_{2}\right)$ in the manner described in [23] with $C_{2}^{\perp} \subset C_{1}$. In this paper, any code pair written in the form $\left(C_{1}, C_{2}\right)$ is supposed to satisfy the constraint $C_{2}^{\perp_{s}} \subset C_{1}$, where $C^{\perp_{s}}$ denotes the $s$-Galois dual of $C$ with $0 \leq s<e$ (see [8, 19]).

There are only few works dealing with CSS construction based on two distinct classical codes [1, 2, 9, 18, 22]. In [1, 2], existence conditions on quantum BCH codes are established, whereas in [18] quantum codes derived from two distinct classical BCH codes are presented. In [9] concatenated quantum codes have been constructed. All of CSS construction are only to $\mathrm{BCH}$ codes or constacyclic codes. Recently, we in [20] proposed two new criteria for the

$C_{2}^{\perp_{s}} \subset C_{1}$ based on $\operatorname{rk}\left(G_{1}\left(G_{2}^{\left(p^{e-s}\right)}\right)^{T}\right)$ or $\operatorname{rk}\left(\begin{array}{c}G_{1} \\ H_{2}^{\left(p^{e-s}\right)}\end{array}\right)$ with $0 \leq s<e$, where $G_{1}$ and $G_{2}$ are generator matrices for $C_{1}$ and $C_{2}$, respectively, and $H_{2}$ is parity-check matrix for the linear code $C_{2}$. Then we gave a class of new quantum codes and some new quantum MDS codes. The aim of this paper is to continue constructing new quantum MDS codes by the methods of ours which used in [20].

This paper organized as follows. In Section 2, we recall the basic definitions and properties of linear codes, $s$-Galois dual codes and quantum codes. In Section 3, using the methods of [20], we obtain two new classes of MDS quantum codes by means of Generalized ReedSolomon (GRS) codes, one of which generalizes a result of [20]. Finally, a brief summary of this work is described in Section 4.

\section{Preliminaries}

In this section, we recall some basic concepts and results about linear codes, $s$-Galois dual codes and quantum codes, necessary for the development of this work. For more details, please refer to $[8,10,19,1]$.

Throughout this paper, let $\mathbb{F}_{q}$ be a finite field with $q=p^{e}$ elements, where $p$ is a prime number and $e \geq 1$ is an integer. For a positive integer $n$, let $\mathbb{F}_{q}^{n}$ denote the vector space of all $n$-tuples over $\mathbb{F}_{q}$. A linear $[n, k]_{q}$ code $C$ over $\mathbb{F}_{q}$ is a $k$-dimensional subspace of $\mathbb{F}_{q}^{n}$. The Hamming weight $w_{H}(\mathbf{c})$ of a codeword $\mathbf{c} \in C$ is the number of nonzero components of c. The Hamming distance of two codewords $\mathbf{c}_{1}, \mathbf{c}_{2} \in C$ is $d_{H}\left(\mathbf{c}_{1}, \mathbf{c}_{2}\right)=w_{H}\left(\mathbf{c}_{2}-\mathbf{c}_{1}\right)$. The minimum Hamming distance $d$ of $C$ is the minimum Hamming distance between any two 
distinct codewords of $C$. An $[n, k, d]_{q}$ code is an $[n, k]_{q}$ code with the minimum Hamming distance $d$.

\section{$2.1 s$-Galois dual codes}

In [8], Fan and Zhang introduce a kind of forms on $\mathbb{F}_{q}^{n}$ as follows ([8, Definition 4.1]).

For each integer $s$ with $0 \leq s<e$, define:

$$
[\mathbf{x}, \mathbf{y}]_{s}=x_{1} y_{1}^{p^{s}}+\cdots+x_{n} y_{n}^{p^{s}}, \quad \forall \mathbf{x}, \mathbf{y} \in \mathbb{F}_{q}^{n}
$$

We call $[\mathbf{x}, \mathbf{y}]_{s}$ the $s$-Galois form on $\mathbb{F}_{q}^{n}$. It is just the usual Euclidean inner product if $s=0$. And, it is the Hermitian inner product if $e$ is even and $s=\frac{e}{2}$. For any code $C$ of $\mathbb{F}_{q}^{n}$, the following code

$$
C^{\perp_{s}}=\left\{\mathbf{x} \in \mathbb{F}_{q}^{n} \mid[\mathbf{c}, \mathbf{x}]_{s}=0, \forall \mathbf{c} \in C\right\}
$$

is called the $s$-Galois dual code of $C$. Note that $C^{\perp_{s}}$ is linear whenever $C$ is linear or not. Then $C^{\perp_{0}}$ (simply, $C^{\perp}$ ) is just the Euclidean dual code of $C$, and $C^{\perp_{\frac{e}{2}}}$ (simply, $C^{\perp_{H}}$ ) is just the Hermitian dual code of $C$. If $C \subset C^{\perp_{s}}$, then $C$ is said to be $s$-Galois self-orthogonal. Moreover, $C$ is said to be $s$-Galois self-dual if $C=C^{\perp_{s}}$.

From the fact that $s$-Galois form is non-degenerate ([8, Remark 4.2]), it follows immediately that $\operatorname{dim}_{\mathbb{F}_{q}} C+\operatorname{dim}_{\mathbb{F}_{q}} C^{\perp_{s}}=n$.

A linear code $C$ is called $s$-Galois dual-containing if $C^{\perp_{s}} \subset C$.

For a $t \times t$ matrix $A=\left(a_{i j}\right)_{t \times t}$ over $\mathbb{F}_{q}$, we denote $A^{\left(p^{e-s}\right)}=\left(a_{i j}^{p^{e-s}}\right)_{t \times t}$. In particular, for a vector $\mathbf{a}=\left(a_{1}, a_{2}, \ldots, a_{n}\right) \in \mathbb{F}_{q}^{n}$, we have

$$
\mathbf{a}^{p^{e-s}}=\left(a_{1}^{p^{e-s}}, a_{2}^{p^{e-s}}, \ldots, a_{n}^{p^{e-s}}\right) .
$$

And for a linear code $C$ of $\mathbb{F}_{q}^{n}$, we define $C^{p^{e-s}}$ to be the set $\left\{\mathbf{a}^{p^{e-s}} \mid \mathbf{a} \in C\right\}$ which is also a linear code. It is easy to see that the $s$-Galois dual $C^{\perp_{s}}$ is equal to the Euclidean dual $\left(C^{p^{e-s}}\right)^{\perp}$ of the linear code $C^{p^{e-s}}$.

\subsection{Quantum codes}

Let $V_{n}$ be the Hilbert space $V_{n}=\mathbb{C}^{q^{n}}=\mathbb{C}^{q} \otimes \cdots \otimes \mathbb{C}^{q}$. Let $|x\rangle$ be the vectors of an orthonormal basis of $\mathbb{C}^{q^{n}}$, where the labels $x$ are elements of $\mathbb{F}_{q}$. Then $V_{n}$ has the following orthonormal basis $\left\{|\mathbf{c}\rangle=\left|c_{1} c_{2} \cdots c_{n}\right\rangle=\left|c_{1}\right\rangle \otimes\left|c_{2}\right\rangle \otimes \cdots \otimes\left|c_{n}\right\rangle: \mathbf{c}=\left(c_{1}, c_{2}, \ldots, c_{n}\right) \in \mathbb{F}_{q}^{n}\right\}$.

Consider $a, b \in \mathbb{F}_{q}$, the unitary linear operators $X(a)$ and $Z(b)$ in $\mathbb{C}^{q}$ are defined by $X(a)|x\rangle=|x+a\rangle$ and $Z(b)|x\rangle=\omega^{\operatorname{tr}(b x)}|x\rangle$, respectively, where $\omega=\exp (2 \pi i / p)$ is a primitive $p$-th root of unity and tr is the trace map from $\mathbb{F}_{q}$ to $\mathbb{F}_{p}$.

Let $\mathbf{a}=\left(a_{1}, \ldots, a_{n}\right) \in \mathbb{F}_{q}^{n}$, we write $X(\mathbf{a})=X\left(a_{1}\right) \otimes \cdots \otimes X\left(a_{n}\right)$ and $Z(\mathbf{a})=Z\left(a_{1}\right) \otimes \cdots \otimes$ $Z\left(a_{n}\right)$ for the tensor products of $n$ error operators. The set $E_{n}=\left\{X(\mathbf{a}) Z(\mathbf{b}): \mathbf{a}, \mathbf{b} \in \mathbb{F}_{q}^{n}\right\}$ is 
an error basis on the complex vector space $\mathbb{C}^{q^{n}}$ and then we set $G_{n}=\left\{\omega^{c} X(\mathbf{a}) Z(\mathbf{b}): \mathbf{a}, \mathbf{b} \in\right.$ $\left.\mathbb{F}_{q}^{n}, c \in \mathbb{F}_{p}\right\}$ is the error group associated with $E_{n}$.

Definition 2.1. A q-qry quantum code of length $n$ is a subspace $Q$ of $V_{n}$ with dimension $K>1$. A quantum code $Q$ of dimension $K>2$ is called quantum code with parameters $((n, K, d))_{q}$ or $[[n, k, d]]_{q}$, where $k=\log _{q} K$ if $Q$ detect $d-1$ quantum digits of errors for $d \geq 1$. Namely, if for every orthogonal pair $|u\rangle,|v\rangle$ in $Q$ with $\langle u \mid v\rangle=0$ and every $e \in G_{n}$ with $W_{Q}(e) \leq d-1,|u\rangle$ and $e|v\rangle$ are orthogonal, i.e., $\langle u|e| v\rangle=0$. Such a quantum code is called pure if $\langle u|e| v\rangle=0$ for any $|u\rangle$ and $|v\rangle$ in $Q$ and any $e \in G_{n}$ with $1 \leq W_{Q}(e) \leq d-1$. A quantum code $Q$ with $K=1$ is always pure.

From the classical linear codes, we can directly obtain a family of quantum codes by using the called CSS given by the following theorem.

Theorem 2.2. ([5, 6, 23]). (CSS Code Construction) Let $C_{1}$ and $C_{2}$ denote two classical linear codes with parameters $\left[n, k_{1}, d_{1}\right]_{q}$ and $\left[n, k_{2}, d_{2}\right]_{q}$, respectively, such that $C_{2}^{\perp} \subset C_{1}$. Then there exists an $\left[\left[n, k_{1}+k_{2}-n, d\right]\right]_{q}$ quantum code where $d=\min \left\{W_{H}(c) \mid c \in\left(C_{1} \backslash C_{2}^{\perp}\right) \cup\right.$ $\left(C_{2} \backslash C_{1}^{\perp}\right\}$ that is pure to $\min \left\{d_{1}, d_{2}\right\}$.

Corollary 2.3. Let $C_{1}$ and $C_{2}$ denote two classical linear codes with parameters $\left[n, k_{1}, d_{1}\right]_{q}$ and $\left[n, k_{2}, d_{2}\right]_{q}$, respectively, such that $C_{2}^{\perp s} \subset C_{1}$. Then there exists an $\left[\left[n, k_{1}+k_{2}-n, d\right]\right]_{q}$ quantum code where $d=\min \left\{W_{H}(c) \mid c \in\left(C_{1} \backslash\left(C_{2}^{p^{e-s}}\right)^{\perp}\right) \cup\left(C_{2}^{p^{e-s}} \backslash C_{1}^{\perp}\right)\right\} \geq \min \left\{d_{1}, d_{2}\right\}$ that is pure to $\min \left\{d_{1}, d_{2}\right\}$.

Proof. Obviously, $C_{2}^{p^{e-s}}$ is also an $\left[n, k_{2}, d_{2}\right]_{q}$ linear code over $\mathbb{F}_{q}$. Since $C_{2}^{\perp s} \subset C_{1}$ is equal to $C_{1}^{\perp} \subset C_{2}^{p^{e-s}}$, there exists an $\left[\left[n, k_{1}+k_{2}-n, d\right]\right]_{q}$ quantum code where $d=$ $\min \left\{W_{H}(c) \mid c \in\left(C_{1} \backslash\left(C_{2}^{p^{e-s}}\right)^{\perp}\right) \cup\left(C_{2}^{p^{e-s}} \backslash C_{1}^{\perp}\right\}\right.$ by Theorem 2.2.

Lemma 2.4. Let $C_{i}$ be an $\left[n, k_{i}\right]_{q}$ linear code over $\mathbb{F}_{q}$ with generator matrix $G_{i}$ for $i=1,2$. Then $\operatorname{rk}\left(G_{1}\left(G_{2}^{\left(p^{e-s}\right)}\right)^{T}\right)=k_{1}-\operatorname{dim}_{F_{q}}\left(C_{1} \cap C_{2}^{\perp s}\right)$.

The following two lemmas characterizes the $s$-Galois dual-containing of two linear codes, the proofs of which can be found in $[20$.

Lemma 2.5. Let $C_{i}$ be an $\left[n, k_{i}\right]_{q^{2}}$ linear code over $\mathbb{F}_{q}$ with generator matrix $G_{i}$ for $i=1,2$. Then $C_{2}^{\perp H} \subset C_{1}$ if and only if $\operatorname{rk}\left(G_{1}\left(G_{2}^{\left(p^{e-s}\right)}\right)^{T}\right) \leq k_{1}+k_{2}-n$ and $k_{1}+k_{2} \geq n$.

Lemma 2.6. Let $C_{i}$ be an $\left[n, k_{i}\right]_{q}$ linear code over $\mathbb{F}_{q}$ with generator matrix $G_{i}$ and paritycheck $H_{i}$ for $i=1,2$. Then $C_{2}^{\perp_{s}} \subset C_{1}$ if and only if $\mathrm{rk}\left(\begin{array}{c}G_{1} \\ H_{2}^{\left(p^{-e s}\right)}\end{array}\right) \leq k_{1}$ and $k_{1}+k_{2} \geq n$.

Combining Lemma 2.5 or Lemma 2.6 and Corollary 2.3, we have the following new methods to construct quantum codes. 
Theorem 2.7. Let $C_{i}$ be an $\left[n, k_{i}\right]_{q}$ linear code over $\mathbb{F}_{q}$ with generator matrix $G_{i}$ or paritycheck matrix $H_{i}$ for $i=1,2$. If $\operatorname{rk}\left(\begin{array}{c}G_{1} \\ H_{2}^{\left(p^{e-s}\right)}\end{array}\right) \leq k_{1}$ or $\operatorname{rk}\left(G_{1}\left(G_{2}^{\left(p^{e-s}\right)}\right)^{T}\right)=k_{1}-\operatorname{dim}_{F_{q}}\left(C_{1} \cap\right.$ $\left.C_{2}^{\perp_{s}}\right) \leq k_{1}+k_{2}-n$ and $k_{1}+k_{2} \geq n$, then there exist a quantum code with parameters $\left[\left[n, k_{1}+k_{2}-n, d\right]\right]_{q}$, where $d \geq \min \left\{d_{1}, d_{2}\right\}$.

To see that a quantum code $Q$ is good in terms of its parameters, we have to introduce the quantum Singleton bound (see [3]).

Theorem 2.8. Let $Q$ be a quantum code with parameters $[[n, k, d]]_{q}$. Then $2 d \leq n-k+2$.

If a quantum code $Q$ with parameters $[[n, k, d]]_{q}$ attains the quantum Singleton bound $2 d=n-k+2$, then it is called a quantum maximum-distance-separable (MDS) code.

\section{$3 \quad$ New quantum MDS codes construction}

In this section, we construct two new classes of quantum MDS codes.

\subsection{Codes construction I}

The hull of $C$ is the code $C \cap C^{\perp}$, denoted by $\operatorname{Hull}(C)$, in the terminology that was introduced in [4].

The following two lemmas is due to Luo, Cao and Chen [17].

Lemma 3.1. Let $q>3$ be an odd prime power. Then

(1) there exists an $[q+1, k, q-k+2]_{q} M D S$ code with $l$-dimensional hull for any $1 \leq l \leq$ $k-1$.

(2) there exists an $\left[q+1, \frac{q+1}{2}, \frac{q+1}{2}+1\right]_{q}$ MDS code with l-dimensional hull for any $1 \leq$ $l \leq \frac{q+1}{2}$.

Lemma 3.2. Let $q=2^{m}$, where $m>1$ is an integer. Then there exists an $[q+1, k, q-k+2]_{q}$ $M D S$ code with $l$-dimensional hull for any $1 \leq l \leq k-1$.

With the above lemmas and Theorem 2.7, the construction of MDS quantum codes turns into that of MDS linear codes with the determined dimensional hull. Then we have the following results.

Theorem 3.3. Let $q>3$ be an odd prime power.

(1) If $\left\lceil\frac{q+2}{2}\right\rceil \leq k \leq q+1$, then there exists an $[[q+1,2 k-q-1, q-k+2]]_{q}$ quantum MDS code.

(2) There exists an $\left[\left[q+1,0, \frac{q+1}{2}+1\right]\right]_{q}$ quantum $M D S$ code. 
Proof. (1) In Lemma 3.1(1), let $q+1-k \leq l \leq k-1$. Then $k \geq\left\lceil\frac{q+2}{2}\right\rceil$. By Lemma 2.4, we have

$$
\operatorname{rank}\left(G G^{T}\right)=k-l \leq 2 k-q-1 .
$$

According to Theorems 2.7 and 2.8 , there exists an $[[q+1,2 k-q-1, q-k+2]]_{q}$ quantum MDS code.

(2) In Lemma 3.1(2), let $l=k=\frac{q+1}{2}$. By Lemma 2.4, we have

$$
\operatorname{rank}\left(G G^{T}\right)=k-l=0 \leq 2 k-q-1 .
$$

According to Theorems 2.7 and 2.8 , there exists an $\left[\left[q+1,0, \frac{q+1}{2}+1\right]\right]_{q}$ quantum MDS code.

The proof of the following theorem is similar to the above theorem and is omitted here.

Theorem 3.4. Let $q=2^{m}$, where $m>1$ is an integer. If $\frac{q+2}{2} \leq k \leq q+1$, then there exists an $[[q+1,2 k-q-1, q-k+2]]_{q}$ quantum MDS code.

Remark 3.5. Fang et al.[13] gave an open problem: if $l=2^{s}>2$, does there exist a quantum MDS code of length $l^{2}+1$ and minimum distance $l$ ? In Theorem 3.4, let $m=2 s$ such that $q=l^{2}$. Taking $k=\frac{l^{2}+2}{2}+\frac{(l-1)^{2}+1}{2}$, then there exists an $\left[\left[l^{2}+1, l^{2}-2 l+3, l\right]\right]_{q}$ quantum MDS code, which is implies that the open problem is right.

Example 1. In Theorems 3.3 and 3.4, taking some special values of $q$, we obtain some quantum MDS codes in Table 1. In fact, Theorems 3.3 and 3.4 include the Theorem 4.2 given in [14].

\subsection{Codes construction II}

Firstly, we review some basic notations and results about generalized Reed-Solomon codes. For the details, the reader is referred to [15]. Let $n$ be a positive integer with $1<n \leq q$, $\alpha_{1}, \ldots, \alpha_{n}$ be $n$ distinct elements of $\mathbb{F}_{q}$, and let $v_{1}, \ldots, v_{n}$ be $n$ nonzero elements of $\mathbb{F}_{q}$. For $k$ between 1 and $n$, the generalized Reed-Solomon code $G R S_{k}(\mathbf{a}, \mathbf{v})$ is defined by

$$
G R S_{k}(\mathbf{a}, \mathbf{v})=\left\{\left(v_{1} f\left(\alpha_{1}\right), \ldots, v_{n} f\left(\alpha_{n}\right)\right) \mid f(x) \in \mathbb{F}_{q}[x], \operatorname{deg}(f(x)) \leq k-1\right\},
$$

where $\mathbf{a}, \mathbf{v}$ denote the vectors $\left(\alpha_{1}, \ldots, \alpha_{n}\right),\left(v_{1}, \ldots, v_{n}\right)$, respectively. Clearly, $G R S_{k}(\mathbf{a}, \mathbf{v})$ has a generator matrix

$$
G=\left(\begin{array}{cccc}
v_{1} & v_{2} & \cdots & v_{n} \\
v_{1} \alpha_{1} & v_{2} \alpha_{2} & \cdots & v_{n} \alpha_{n} \\
v_{1} \alpha_{1}^{2} & v_{2} \alpha_{2}^{2} & \cdots & v_{n} \alpha_{n}^{2} \\
\vdots & \vdots & \cdots & \vdots \\
v_{1} \alpha_{1}^{k-1} & v_{2} \alpha_{2}^{k-1} & \cdots & v_{n} \alpha_{n}^{k-1}
\end{array}\right)
$$


Table 1: New quantum MDS codes

\begin{tabular}{c|c|c}
\hline$q$ & New quantum MDS codes & quantum MDS codes from Theorem 4.2 in [14] \\
\hline 9 & {$[[10,2,5]]_{9},[[10,4,4]]_{9},[[10,6,3]]_{9}$,} & {$[[10,8,2]]_{9},[[10,6,3]]_{9},[[10,4,4]]_{9}$,} \\
& {$[[10,8,2]]_{9},[[10,10,1]]_{25},[[10,0,6]]_{9}$} & \\
\hline 16 & {$[[17,1,9]]_{16},[[17,3,8]]_{16},[[17,5,7]]_{16}$,} & {$[[17,17,1]]_{16},[[17,15,2]]_{16},[[17,13,3]]_{16}$,} \\
& {$[[17,7,6]]_{16},[[17,9,5]]_{16},[[17,11,4]]_{16}$,} & {$[[17,11,4]]_{16},[[17,9,5]]_{16}$,} \\
& {$[[17,13,3]]_{16},[[17,15,2]]_{16},[[17,17,1]]_{16}$} & \\
\hline 25 & {$[[26,2,13]]_{25},[[26,4,12]]_{25},[[26,6,11]]_{25}$,} & {$[[26,16,6]]_{25},[[26,18,5]]_{25},[[26,20,4]]_{25}$,} \\
& {$[[26,8,10]]_{25},[[26,10,9]]_{25},[[26,12,8]]_{25}$} & {$[[26,22,3]]_{25},[[26,24,2]]_{25},[[26,26,1]]_{25}$} \\
& {$[[26,14,7]]_{25},[[26,16,6]]_{25},[[26,18,5]]_{25}$,} & \\
& {$[[26,20,4]]_{25},[[26,22,3]]_{25},[[26,24,2]]_{25}$,} & \\
& {$[[26,26,1]]_{25},[[26,0,14]]_{25}$} & \\
\hline
\end{tabular}

It is well known that the code $G R S_{k}(\mathbf{a}, \mathbf{v})$ is an $[n, k, n-k+1]_{q}$ MDS code.

The following result can be found in [15].

Lemma 3.6. Let $\mathbf{1}$ be all-one word of length $n$. The dual code of $G R S_{k}(\mathbf{a}, \mathbf{1})$ is $G R S_{n-k}(\mathbf{a}, \mathbf{u})$, where $\mathbf{u}=\left(u_{1}, u_{2}, \ldots, u_{n}\right)$ with $u_{i}=\prod_{1 \leq j \leq n, j \neq i}\left(\alpha_{i}-\alpha_{j}\right)^{-1}$.

Theorem 3.7. Let $n \leq q, 1 \leq k_{1} \leq n$ and $1 \leq k_{2} \leq n$. If $k_{1}+k_{2} \geq n$, then

(1) there exists a quantum code with parameters $\left[\left[n, k_{1}+k_{2}-n, d\right]\right]_{q}$, where $d \geq \min \{n-$ $\left.k_{1}+1, n-k_{2}+1\right\}$

(2) when $k_{1}=k_{2}=k$ and $k \geq\left\lceil\frac{n}{2}\right\rceil$, there exists a quantum MDS code with parameters $[[n, 2 k-n, n-k+1]]_{q}$.

Proof. (1) Let $\mathbf{u}=\left(u_{1}, u_{2}, \ldots, u_{n}\right)$, where $u_{i}=\prod_{1 \leq j \leq n, j \neq i}\left(\alpha_{i}-\alpha_{j}\right)^{-1}$ for $1 \leq i \leq n$. It is well known that $G R S_{k_{1}}(\mathbf{a}, \mathbf{u})$ has a generator matrix

$$
G_{1}=\left(\begin{array}{cccc}
u_{1} & u_{2} & \cdots & u_{n} \\
u_{1} \alpha_{1} & u_{2} \alpha_{2} & \cdots & u_{n} \alpha_{n} \\
u_{1} \alpha_{1}^{2} & u_{2} \alpha_{2}^{2} & \cdots & u_{n} \alpha_{n}^{2} \\
\vdots & \vdots & \cdots & \vdots \\
u_{1} \alpha_{1}^{k_{1}-1} & u_{2} \alpha_{2}^{k_{1}-1} & \cdots & u_{n} \alpha_{n}^{k_{1}-1}
\end{array}\right) .
$$


Table 2: A comparison of new quantum MDS codes

\begin{tabular}{c|c|c|c}
\hline$q$ & Length $n$ & Distance $d$ & Distance $d^{\prime}$ in Refs. \\
\hline$l^{2}$ & $n=l^{2}$ & $2 \leq d \leq l^{2}-\left\lfloor\frac{l^{2}}{2}\right\rfloor+1$ & $3 \leq d^{\prime} \leq l+1[16]$ \\
\hline$l^{2}$ & $n=l^{2}-1$ & $2 \leq d \leq l^{2}-\left\lfloor\frac{l^{2}-1}{2}\right\rfloor$ & $3 \leq d^{\prime} \leq l-1[16]$ \\
\hline$l^{2}$ & $n=1+\frac{r\left(l^{2}-1\right)}{s}$ & $2 \leq d \leq a-b+2$ & \\
& $s \mid(l-1)$ and $1 \leq r \leq s$ & $a=\frac{r\left(l^{2}-1\right)}{s}, b=\left\lfloor\frac{s+r\left(l^{2}-1\right)}{2 s}\right\rfloor$ & $2 \leq d^{\prime} \leq \frac{r(l-1)}{s}+1[12]$ \\
\hline$l^{2}$ & $n=t l, 1 \leq t \leq l$ & $2 \leq d \leq t l-\left\lfloor\frac{t l}{22}\right\rfloor+1$ & $2 \leq d^{\prime} \leq\left\lfloor\frac{t l+l-1}{l+1}\right\rfloor+1[13]$ \\
\hline$l^{2}$ & $n=\lambda(l+1), \lambda$ is an odd & & $2 \leq d \leq \frac{\lambda(l+1)}{2}+1$ \\
& divisor of $l-1$ & $2 \leq d^{\prime} \leq \frac{l+1}{2}+\lambda[21]$ \\
\hline
\end{tabular}

By Lemma 3.6, $G R S_{k_{2}}(\mathbf{a}, \mathbf{1})$ has a parity check matrix

$$
H_{2}=\left(\begin{array}{cccc}
u_{1} & u_{2} & \cdots & u_{n} \\
u_{1} \alpha_{1} & u_{2} \alpha_{2} & \cdots & u_{n} \alpha_{n} \\
u_{1} \alpha_{1}^{2} & u_{2} \alpha_{2}^{2} & \cdots & u_{n} \alpha_{n}^{2} \\
\vdots & \vdots & \cdots & \vdots \\
u_{1} \alpha_{1}^{n-k_{2}-1} & u_{2} \alpha_{2}^{n-k_{2}-1} & \cdots & u_{n} \alpha_{n}^{n-k_{2}-1}
\end{array}\right) .
$$

If $k_{1}+k_{2} \geq n$, i.e., $k_{1}-1 \geq n-k_{2}-1$, then $\operatorname{rk}\left(\begin{array}{c}G_{1} \\ H_{2}^{\left(p^{e-0}\right)}\end{array}\right)=k_{1} \leq k_{1}$. Thus, by Theorem 2.7, there exists a quantum code with parameters $\left[\left[n, k_{1}+k_{2}-n, d\right]\right]_{q}$, where $d \geq \min \left\{n-k_{1}+1, n-k_{2}+1\right\}$.

(2) When $k_{1}=k_{2}=k$, then by $k_{1}+k_{2} \geq n$, we have $k \geq\left\lceil\frac{n}{2}\right\rceil$. Note that $d \geq$ $\min \left\{n-k_{1}+1, n-k_{2}+1\right\}=n-k+1$.

On the other hand, by Theorem $2.8,2 d \leq n-(2 k-n)+2=2(n-k+1)$, i.e., $d \leq n-k+1$.

It follows that $d=n-k+1$, which implies that there exists a quantum MDS code with parameters $[[n, 2 k-n, n-k+1]]_{q}$.

Remark 3.8. Theorem 3.7 generalizes a result of [20]. In the above Theorem [3.7, when $n \mid q-1$ and $n-k=1+j$ we can obtain Theorem 4.11 in [20].

Example 2. In Theorem 3.7, let $q=l^{2}$ where l is a prime power. In Table 2, we compare our quantum MDS codes with previously known quantum MDS codes available in [12], [13], [16] and [21]. As can be seen, when the code length $n$ is fixed, the new quantum MDS codes have much larger minimum distance. 


\section{Conclusion}

In this paper, by using two methods and generalized Reed-Solomon codes, we have constructed two new families of quantum MDS codes with flexible parameters. We see that a result of [20] is special case of ours and the parameters of some previous results are also improved. Moreover, we give an affirmative answer to the open problem raised by Fang et al. in [13].

Acknowledgements This work was supported by Scientific Research Foundation of Hubei Provincial Education Department of China(Grant No. Q20174503) and the National Science Foundation of Hubei Polytechnic University of China (Grant No.12xjz14A and 17xjz03A).

\section{References}

[1] Aly, S.A., Klappenecker, A., Sarvepalli, P.K.: On quantum and classical BCH codes, IEEE Trans. Inf. Theory 53(3), 1183-1188(2007)

[2] Aly, S.A., Klappenecker, A., Sarvepalli, P.K.: Primitive quantum BCH codes over finite fields, in:Proc.Int.Symp.Inform.Theory, ISIT,1114-1118(2006)

[3] Ashikhmin, A., Knill, E.: Nonbinary quantum stabilizer codes, IEEE Trans. Inf. Theory 47, 3065-3072 (2001).

[4] Assmus, Jr.E.F., Key, J.: Designs and Their Codes. Cambridge University Press, Cambridge (1992). Cambridge Tracts in Mathematics, vol.103 (Second printing with corrections, 1993)

[5] Calderbank, A.R., Rains, E.M., Shor, P.W., Sloane, N.J.A.: Quantum error correction via codes over GF(4), IEEE Trans. Inf. Theory 44(4), 1369-1387(1998)

[6] Calderbank, A.R., Rains, E.M., Shor, P.W., Sloane, N.J.A.: Quantum error correction and orthogonal geometry, Phys. Rev. Lett. 78(3), 405-408(1997)

[7] Calderbank, A.R., Shor, P.W.: Good quantum error correcting codes exist, Phys. Rev. A, 54,1098-1105(1996)

[8] Fan, Y., Zhang, L.: Galois self-dual constacyclic codes, Des. Codes Cryptogr. 84, 473$492(2017)$

[9] Hamada, M.: Concatenated quantum codes constructible in polynomial time: efficient decoding and error correction, IEEE Trans. Inform. Theory 54(12), 5689-5704(2008) 
[10] Huffman, W.C., Pless, V.: Fundamentals of Error-Correcting Codes, Cambridge University Press, Cambridge(2003)

[11] Hurley, T., Hurley, D.: Coding theory: the unit-derived methodology, Int. J. Information and Coding Theory 5(1), 55-80(2018)

[12] Fang, W., Fu, F.: Some New constructions of quantum MDS codes, IEEE Trans. Inf. Theory 65(12), 7840-7847(2019)

[13] Fang, W., Fu, F.: Two new classea of quantum MDS codes, Finite Fields Appl. 53, 85-98(2018)

[14] Jin, L., Xing, C.: A construction of new quantum MDS codes, IEEE Trans. Inf. Theory 60,2921-2925(2014)

[15] Jin, L., Xing, C.: New MDS self-dual codes from Generalized Reed-Solomon codes, IEEE Trans. Inf. Theory 63,1434-1438(2017)

[16] Jin, L., Ling, S., Luo, J.:Application of classical Hermitian self-orthogonal MDS codes to quantum MDS codes, IEEE Trans. Inf. Theory 56(9),4735-4740(2010)

[17] Luo, G., Cao, X., Chen, X.: MDS codes with arbitrary dimensional hull and their applications, IEEE Trans. Inf. Theory 68(8), 2944-2952(2019)

[18] La Guardia, G.G., Palazzo Jr, R.: Constructions of new families of nonbinary CSS codes, Discrete Mathematics 310, 2935-2945(2010)

[19] Liu ,X., Fan, Y., Liu, H.: Galois LCD codes over finite fields, Finite Field Appl. 49, $227-242(2018)$

[20] Liu, X., Hu, P.: New quantum codes from two linear codes. Quantum Inf Process 19, 78 (2020). https://doi.org/10.1007/s11128-020-2575-0

[21] Kai, X., Zhu, S., Li, P.: Constacyclic codes and some new quantum MDS codes. IEEE Trans. Inf. Theory 60(4), 2080-2086 (2014)

[22] Ma, Z., Lu, X., Feng, K., Feng, D.: On non-binary quantum BCH codes, Lecture Notes in Comput. Sci. 3959 675-683(2006)

[23] Steane, A.M.: Enlargement of Calderbank-Shor-Steane quantum codes, IEEE Trans. Inform. Theory 45(7),2492-2495(1999) 